\title{
ON THE INTERACTION BETWEEN FORCED AND PARAMETRIC OSCILLATIONS IN A SYSTEM WITH TWO DEGREES OF FREEDOM
}

\author{
Tran Kim Chi - NGUYen Van Dinh \\ Institute of Mechanics, Hanoi Vietnam
}

In [1], the interaction between forced and parametric oscillations in a quasi-linear oscillating system with two degrees' of freedom has been studied: both mentioned oscillations occur in the mode $(y)$; the forced one results from an external excitation in the fundamental resonance, directly acting on ( $y$ ); the parametric oscillation (by means of nonlinearities) is indirectly excited by the forced non-resonant oscillation of the mode $(x)$. It has been shown that if the indirectly excited parametric oscillation is very intense, the resonance curve consists of three branches - the left and right of "hyperbolic" form and the middle of "parabolic" form.

In the present paper, the so called "harmonic and parametric case" [1, pp. 335-341] will be examined. Critical singular points [2] will be used to classify different forms of the resonance curve. The case in which the indirectly - excited parametric oscillation is not very intense will be analyzed in detail. As it will be shown, the resonance curve has either a loop or an oval.

1. System under consideration

Let us consider a the quasilinear oscillating system with two degrees of freedom described by the differential equations [1]:

$$
\begin{aligned}
\ddot{x}+\lambda^{2} x+\varepsilon \lambda^{2}\left(h_{0} \dot{x}+\alpha x^{3}+c y^{2} x\right) & =Q \sin \nu t, \\
\ddot{y}+y+\varepsilon\left(h \dot{y}+\beta y^{3}+b x^{2} y\right) & =\varepsilon p \cos (\nu t+\delta) .
\end{aligned}
$$

For simplicity, $h_{0}, \beta$ and $b$ are assumed to be positive.

The oscillations are found in the forms:

$$
\begin{aligned}
& x=q \sin \nu t+a_{1} \cos \left(\lambda t+\psi_{1}\right) ; \quad \dot{x}=\nu q \cos \nu t-\lambda a_{1} \sin \left(\lambda t+\psi_{1}\right) \\
& y=a \cos (\nu t+\psi), \quad \dot{y}=\sim \nu a \sin (\nu t+\psi), \quad q=\frac{Q}{\lambda^{2}-\nu^{2}}
\end{aligned}
$$

and the averaged differential equations in the first approximation are:

$$
\begin{aligned}
\dot{a}_{1} & =-\frac{\varepsilon \lambda}{2} h_{0} a_{1}, \\
\dot{a} & =-\frac{\varepsilon \nu}{2} f_{0}=-\frac{\varepsilon \nu}{2}\left\{\nu h a+\frac{b}{4} q^{2} a \sin 2 \psi+p \sin (\psi-\delta)\right\}, \\
a \dot{\psi} & =\frac{\varepsilon \nu}{2} g_{0}=\frac{\varepsilon \nu}{2}\left\{\left(\frac{3}{4} \beta a^{2}+\frac{b}{2} q^{2}+\Delta\right) a-\frac{b}{4} q^{2} a \cos 2 \psi-p \cos (\psi-\delta)\right\},
\end{aligned}
$$

where $\varepsilon \Delta=\left(1-\nu^{2}\right)$. 

$\frac{b}{4} q^{2}$

Evidently, the intensity of the indirect parametric excitation is characterized by the coefficient

Stationary oscillations are determined from the equations:

$$
a_{1}=0, \quad f_{0}=0, \quad g_{0}=0,
$$

among them, the last two ones can be replaced by their equivalents:

$$
\begin{aligned}
& f=f_{0} \sin \psi-g_{0} \cos \psi=\nu h a \sin \psi-\left[\left(\frac{3}{4} \beta a^{2}+\frac{b}{2} q^{2}+\Delta\right)-\frac{b}{4} q^{2}\right] a \cos \psi+p \cos \delta=0 \\
& g=f_{0} \cos \psi+g_{0} \sin \psi=\left[\left(\frac{3}{4} \beta a^{2}+\frac{b}{2} q^{2}+\Delta\right)+\frac{b}{4} q^{2}\right] a \sin \psi+\nu h a \cos \psi-p \sin \delta=0 .
\end{aligned}
$$

The resonance $C$ (frequency-amplitude characteristic is defined as the ensemble of representing points $\left(\nu^{2}, a\right)$ whose ordinate $a$ is the amplitude of stationary oscillations corresponding to the frequency $\nu$. In general, $C$ consists of two parts: the ordinary part $C_{1}$ and the critical one $C_{2}$.

2. System without damping in the mode $y$

We shall first examine the system without damping in the mode $(y)$. In this case $h=0$ and the system of equations (1.5) becomes simple:

$$
\begin{aligned}
& {\left[\left(\frac{3}{4} \beta a^{2}+\frac{b}{2} q^{2}+\Delta\right)-\frac{b}{4} q^{2}\right] a \cos \psi=p \cos \delta} \\
& {\left[\left(\frac{3}{4} \beta a^{2}+\frac{b}{2} q^{2}+\Delta\right)+\frac{b}{4} q^{2}\right] a \sin \psi=p \sin \delta}
\end{aligned}
$$

In the ordinary region where:

$$
D_{0}=\left[\left(\frac{3}{4} \beta a^{2}+\frac{b}{2} q^{2}+\Delta\right)-\frac{b}{4} q^{2}\right]\left[\left(\frac{3}{4} \beta a^{2}+\frac{b}{2} q^{2}+\Delta\right)+\frac{b}{4} q^{2}\right] \neq 0
$$

from (2.1), $\sin \psi$ and $\cos \psi$ can be calculated without difficulty and the ordinary part $C_{1}$ is easily obtained:

$$
W_{1}\left(\nu^{2}, a^{2}\right)=\frac{p^{2} \cos ^{2} \delta}{\left[\left(\frac{3}{4} \beta a^{2}+\frac{b}{2} q^{2}+\Delta\right)-\frac{b}{4} q^{2}\right]^{2} a^{2}}+\frac{p^{2} \sin ^{2} \delta}{\left[\left(\frac{3}{4} \beta a^{2}+\frac{b}{2} q^{2}+\Delta\right)+\frac{b}{4} q^{2}\right]^{2} a^{2}}-1=0 .
$$

The critical region is characterized by the equality:

$$
D_{0}=\left(\frac{3}{4} \beta a^{2}+\frac{b}{2} q^{2}+\Delta\right)^{2}-\left(\frac{b}{4} q^{2}\right)^{2}=0
$$

It presents two curve in the plane $\left(\nu^{2}, a^{2}\right)$

$$
\begin{aligned}
& \frac{3}{4} \beta a^{2}=\left(\nu^{2}-1\right)-\frac{b}{2} q^{2}-\frac{b}{4} q^{2} \\
& \frac{3}{4} \beta a^{2}=\left(\nu^{2}-1\right)-\frac{b}{2} q^{2}+\frac{b}{4} q^{2} .
\end{aligned}
$$

Along (2.5a), the system (2.1) becomes:

$$
-\frac{b}{2} q^{2} a \cos \psi=p \cos \delta, \quad O a \sin \psi=p \sin \delta .
$$


If $\delta=0, \pi$, the system (2.6) has as solutions:

$$
\begin{aligned}
& \psi=\arccos \frac{ \pm 2 p}{b q^{2} a}, \\
& a^{2} \geq \frac{4 p^{2}}{b^{2} q^{4}} .
\end{aligned}
$$

Analogously, along (2.5b), if $\delta=\frac{\pi}{2}, \frac{3 \pi}{2}$, under the same restriction $(2.8)$ we obtain

$$
\psi= \pm \operatorname{arc} \sin \frac{2 p}{b q^{2} a} .
$$

Thus, for the system without damping in the mode $(y)$, the critical part $C_{2}$ :

- does not exist if $\delta \neq 0, \frac{\pi}{2}, \pi, \frac{3 \pi}{2}$ (the resonance curve consists only of the ordinary part $C_{1}$ );

- is the semi curve given by $(2.5 \mathrm{a})$ bounded below by $(2.8)$ if $\delta=0, \pi$;

- is the semi curve (2.5b) (2.8) if $\delta=\frac{\pi}{2}, \frac{3 \pi}{2}$.

The forms of the resonance curve in the case $h=0$, shown in Figs 1, 2, correspond to $\delta=0$ ( $C_{2}$ exists), $\delta=\frac{\pi}{4}\left(C_{2}\right.$ does not exists) respectively. The case $h=0, \delta=0$ has been presented in [1].

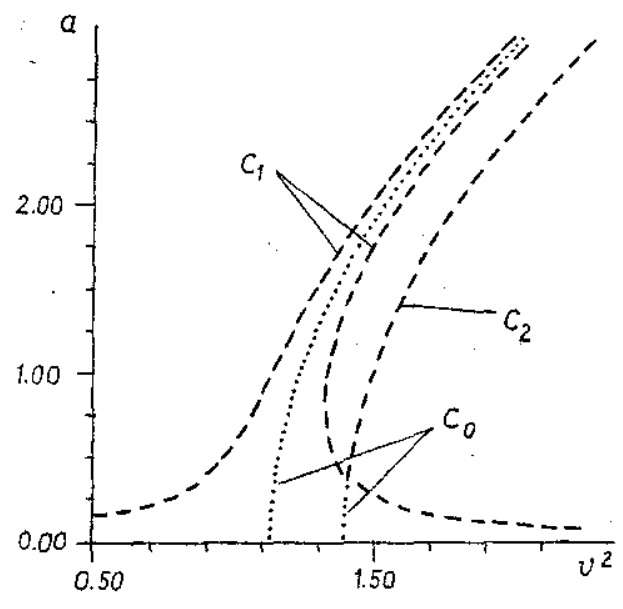

Fig.1

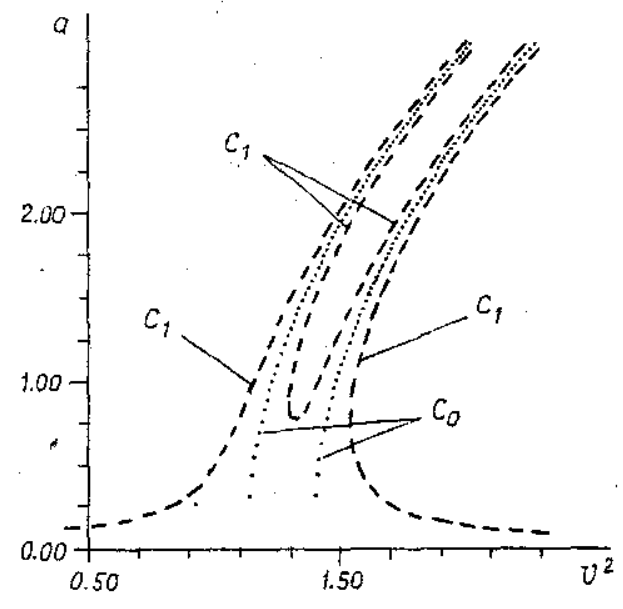

Fig. 2

3. System with damping - Ordinary and critical stationary oscillations

In this case $h>0$, the critical part $C_{2}$ is reduced into an unique point $I$ and various forms of the resonance curve can be obtained.

By $\bar{D}_{0}, \bar{D}_{1}, \bar{D}_{2}$ and $D_{0}, D_{1}, D_{2}$ we denote the following determinants

$$
D_{0}=\left|\begin{array}{cc}
\bar{D}_{0}=a^{2} D_{0} \\
\nu h & -\left[\left(\frac{3}{4} \beta a^{2}+\frac{b}{2} q^{2}+\Delta\right)-\frac{b}{4} q^{2}\right] \\
{\left[\left(\frac{3}{4} \beta a^{2}+\frac{b}{2} q^{2}+\Delta\right)+\frac{b}{4} q^{2}\right]} & \nu h
\end{array}\right|,
$$




$$
\begin{array}{ll}
\bar{D}_{1}=a p D_{1}, & D_{1}=\left|\begin{array}{ccc}
-\cos \delta & -\left[\left(\frac{3}{4} \beta a^{2}+\frac{b}{2} q^{2}+\Delta\right)-\frac{b}{4} q^{2}\right] \\
\sin \delta &
\end{array}\right|, \\
\bar{D}_{2}=a p D_{2}, & D_{2}=\mid\left[\begin{array}{lc}
\left.\left(\frac{3}{4} \beta a^{2}+\frac{b}{2} q^{2}+\Delta\right)+\frac{b}{4} q^{2}\right] & -\cos \delta \\
\sin \delta
\end{array} \mid .\right.
\end{array}
$$

In the ordinary region, where:

$$
D_{0}=\left[\left(\frac{3}{4} \beta a^{2}+\frac{b}{2} q^{2}+\Delta\right)+\frac{b}{4} q^{2}\right]\left[\left(\frac{3}{4} \beta a^{2}+\frac{b}{2} q^{2}+\Delta\right)-\frac{b}{4} q^{2}\right]+\nu^{2} h^{2} \neq 0,
$$

from (1.5), we can calculate $\sin \psi, \cos \psi$ and the ordinary part $C_{1}$ is given by:

$$
W_{1}\left(\nu^{2}, a\right)=\frac{p^{2}\left(D_{1}^{2}+D_{2}^{2}\right)}{a^{2} D_{0}^{2}}-1=0
$$

The critical region is characterized by the equality:

$$
D_{0}=0 \quad \text { or } \quad \frac{3}{4} \beta a^{2}=\left(\nu^{2}-1\right)-\frac{b}{2} q^{2} \pm \sqrt{\left(\frac{b}{4} q^{2}\right)^{2}-h^{2} \nu^{2}} \text {. }
$$

It presents the resonance curve $C_{0}$ of the "pure" indirectly - excited parametric oscillations ( $p=0$, the mode $y$ is not directly excited by the external excitation $\varepsilon p \cos (\nu t+\delta))$.

The critical part $C_{2}$ (of the resonance curve $C$ ) is determined from the equations

$$
D_{0}=0, \quad D_{1}=0, \quad D_{2}=0
$$

under the restrictions:

$$
\begin{array}{r}
a^{2}\left\{\nu^{2} h^{2}+\left[\left(\frac{3}{4} \beta a^{2}+\frac{b}{q} q^{2}+\Delta\right)-\frac{b}{4} q^{2}\right]^{2}\right\} \geq p^{2} \cos ^{2} \delta \\
a^{2}\left\{\nu^{2} h^{2}+\left[\left(\frac{3}{4} \beta a^{2}+\frac{b}{q} q^{2}+\Delta\right)+\frac{b}{4} q^{2}\right]^{2}+\nu^{2} h^{2}\right\} \geq p^{2} \sin ^{2} \delta .
\end{array}
$$

From (3.5) we may obtain an unique compatible point $I_{*}$ of coordinates $\left(\nu_{*}^{2}, a_{*}\right)$ :

$$
\begin{aligned}
& \nu_{*}=\frac{-1}{h} \frac{b}{4} q^{2} \sin 2 \delta, \\
& \frac{3}{4} \beta a_{*}^{2}=\left(\nu_{*}^{2}-1\right)-\frac{b}{2} q^{2}-\frac{b}{4} q^{2} \cos 2 \delta>0
\end{aligned}
$$

at which, the restrictions (3.6) lead to an unique inequality:

$$
a_{*}^{2} \geq a_{c}^{2}=\frac{4 p^{2}}{b^{2} q^{4}} .
$$

Thus: - if (3.8) is satisfied, $I_{*}$ corresponds to critical stationary oscillations,

- if (3.8) is not satisfied, $I_{*}$ does not correspond to any stationary oscillation, $I_{*}$ does not belong to the resonance curve.

By rejecting those (compatible) points satisfying (3.5) but not (3.6), the "whole" resonance curve $C\left(C_{1}+C_{2}\right)$ can be found from the relationship:

$$
W\left(\nu^{2}, a^{2}\right)=p^{2}\left(D_{1}^{2}+D_{2}^{2}\right)-a^{2} D_{0}^{2}=0 .
$$


By $D$, we denote the discriminant:

$$
D=\left(\frac{\partial^{2} W}{\partial \nu^{2} \partial a}\right)^{2}-\left(\frac{\partial^{2} W}{\partial \nu^{2} \partial \nu^{2}}\right) \cdot\left(\frac{\partial^{2} W}{\partial a \partial a}\right)=\frac{3 \beta h a^{2}}{2 \nu_{*}}\left(b^{2} q^{4} a_{*}^{2}-4 p^{2}\right)
$$

- if $D>0$ i.e. $a_{*}^{2}>a_{c}^{2}=\frac{4 p^{2}}{b^{2} q^{4}}, I_{*}$ is a nodal point,

- if $D<0$ i.e. $a_{*}^{2}<a_{c}^{2}, I_{*}$ is an isolated point.

The case $D=0$ i.e. $a_{*}^{2}=a_{c}^{2}$ corresponds either to a returning point or a degenerated nodal point (with double tangent).

\section{Different forms of the resonance curve}

Depending on the intensity of the indirectly - excited parametric oscillation (i.e. on the rate between $h$ and $\frac{b}{4} q^{2}$ ) and on that of the direct external excitation as well as on the dephase $\delta$, different forms of the resonance curve can be obtained.

If the indirectly - excited parametric oscillation does not exists (large $h$, small $\frac{b}{4} q^{2}$ ), the critical region $C_{0}$ lies under the axis $\nu^{2}$. The resonance curve is similar to that of the pure forced one except - it may exist - $\mathrm{a}$ "crevasse" at the top. In Fig. 3 for $h=0.0915 ; p=0.11759, \frac{b}{4} q^{2}=0.1$; $\frac{3}{4} \beta=0.1$, the resonance curves (1), (2) correspond to $\delta=\frac{\pi}{2}, \frac{3 \pi}{4}$, respectively.

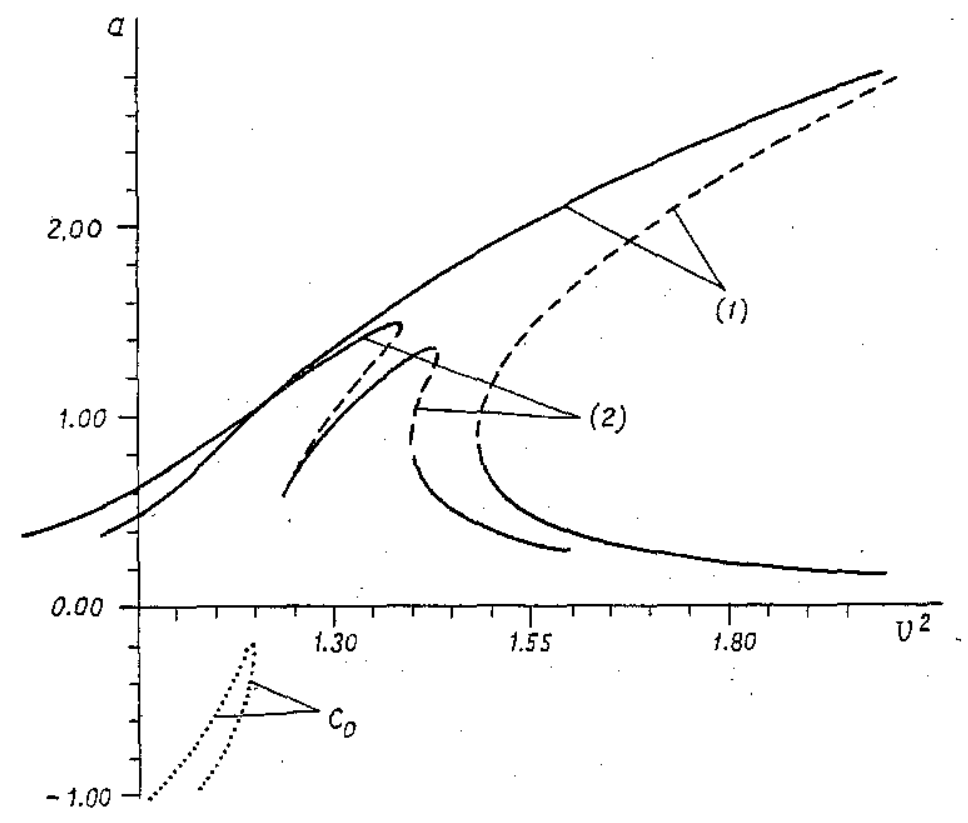

Fig. 8

We shall investigate in detail the case in which the indirectly-excited parametric oscillation is intense enough so that the top of $C_{0}$ lies above the axis $\nu^{2}$.

For small $p$, the resonance curve consists of two branches-the upper $C^{\prime}$ and the lower $C^{\prime \prime}$-lying above and under $C_{0}$ respectively. If $a_{*}^{2}>a_{c}^{2}, I_{*}$ is a representing point, $C^{\prime}$ and $C^{\prime \prime}$ are joined at $I_{*}$, the resonance curve has a loop (Figs $4(\mathrm{a}, \mathrm{b})$ ); if $a_{*}^{2}<a_{c}^{2}, C^{\prime}$ and $C^{\prime \prime}$ are separated by $C_{0}$, the resonance curve has an oval (Figs $5(a, b, c)$ ). 
Increasing $p$, the upper branch moves up while the lower one becomes narrower. In the case of the loop, as $p$ reaches the value $\frac{1}{2} b q a_{*}^{2}$, the loop disappears, the nodal point $I_{*}$ changes into a returning point (Fig. 4c). At certain value $p$, the oval disappears, too (Fig. 5d).

Increasing $p$, further the resonance curve is similar to that of the case without indirectly excited parametric oscillation (Figs $4 \mathrm{~d}, 5 \mathrm{~d}$ ).

In Figs $4(\mathrm{a}, \mathrm{b}, \mathrm{c}, \mathrm{d})$ for $\delta=\frac{3 \pi}{4} ; h=0.09, \frac{b}{4} q^{2}=0.1 ; \frac{3}{4} \beta=0.1$, the resonance curves correspond to $p=0.03 ; 0.05 ; 0.11759 ; 0.15$ respectively.

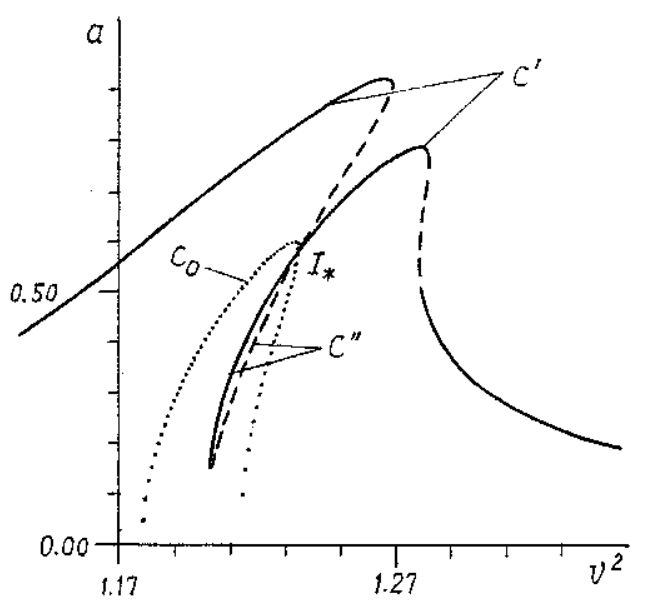

a)

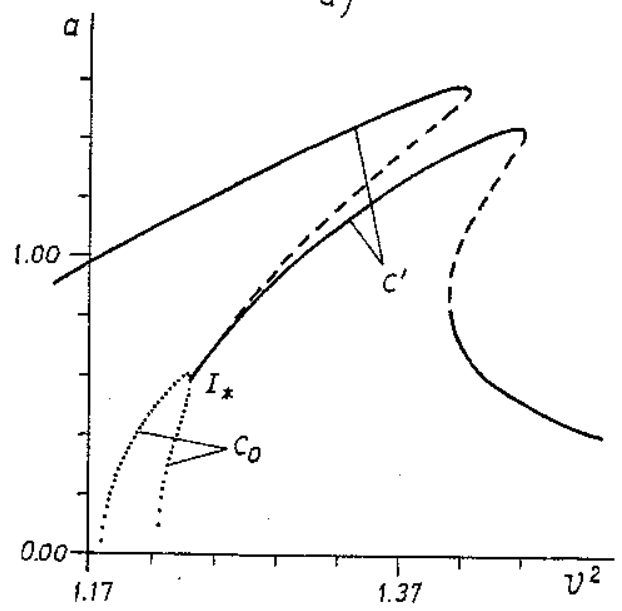

c)

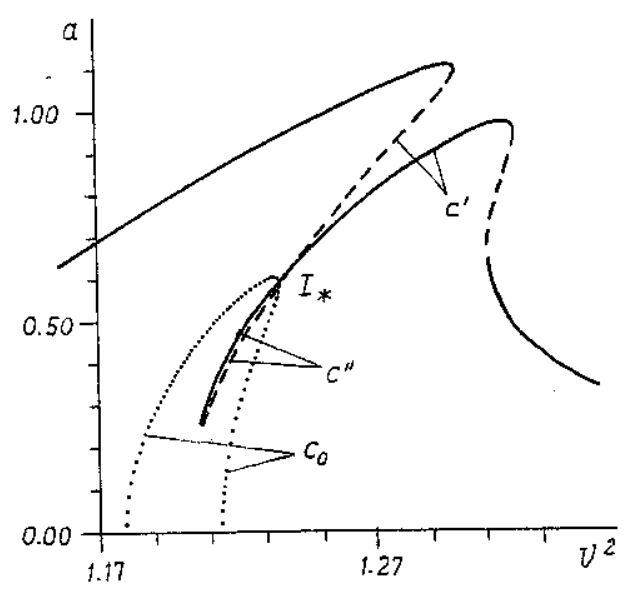

b)

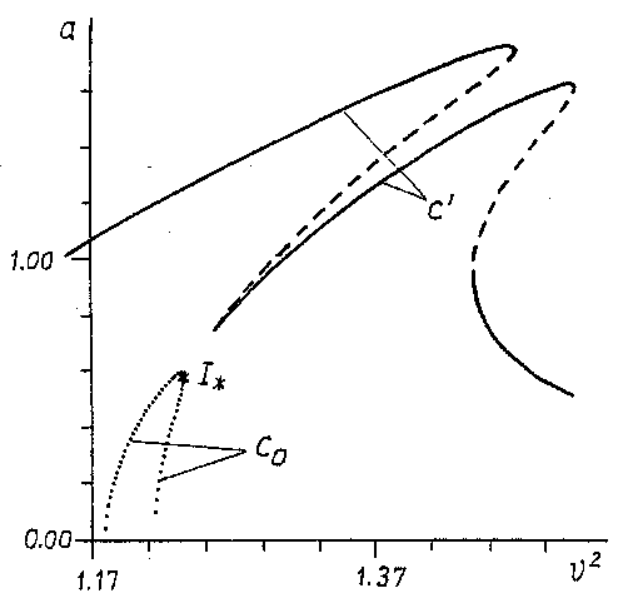

d)

Fig. 4

In Figs $5(\mathrm{a}, \mathrm{b}, \mathrm{c}, \mathrm{d})$ for $\delta=\frac{2 \pi}{3} ; h=0.0877 ; \frac{b}{4} q^{2}=0.1 ; \frac{3}{4} \beta=0.1$ the resonance curves correspond to $p=0.004,0.005 ; 0.006 ; 0.007$.

If the indirectly - excited parametric oscillation is very intense, the top of $C_{0}$ goes up to "infinity" (out of the acceptable region), $C_{0}$ is represented by two nearly parallel curves. For small $p$, the resonance curve $C$ consists of three branches - the left and right of "hyperbolic" form and the middle of "parabolic" form. Increasing $p$, the middle brancher moves up then disappears, the resonance curve has two branches - the left and the right. The resonance curve in this case is of form shown in Fig. 6 and given in [1]. 


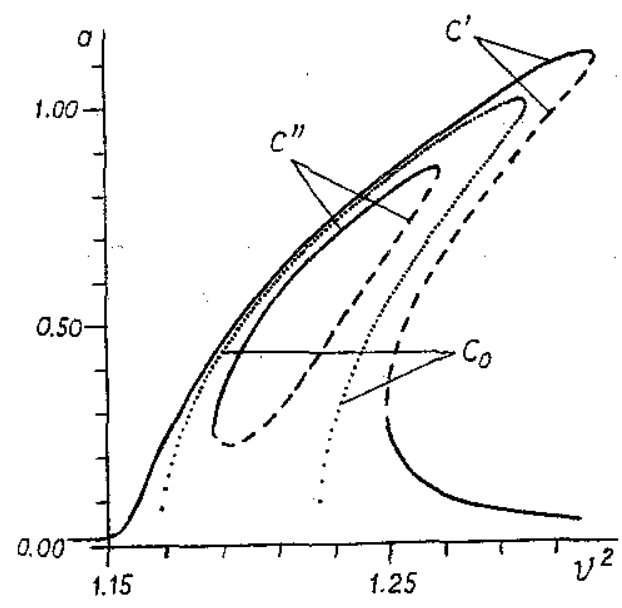

a)

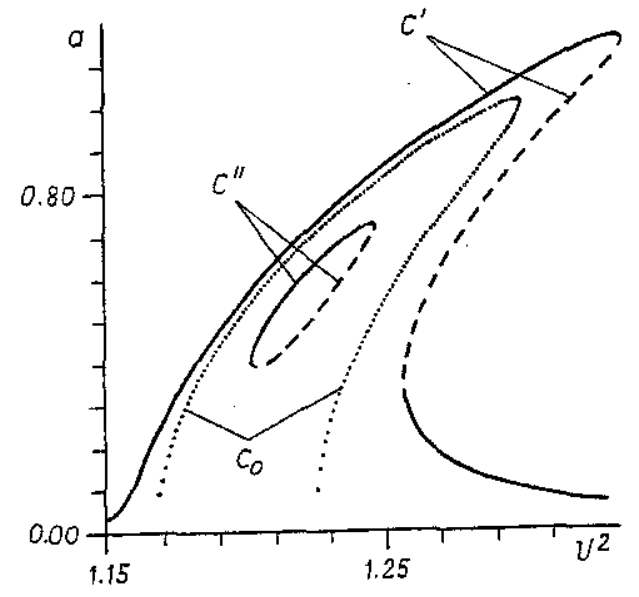

c)

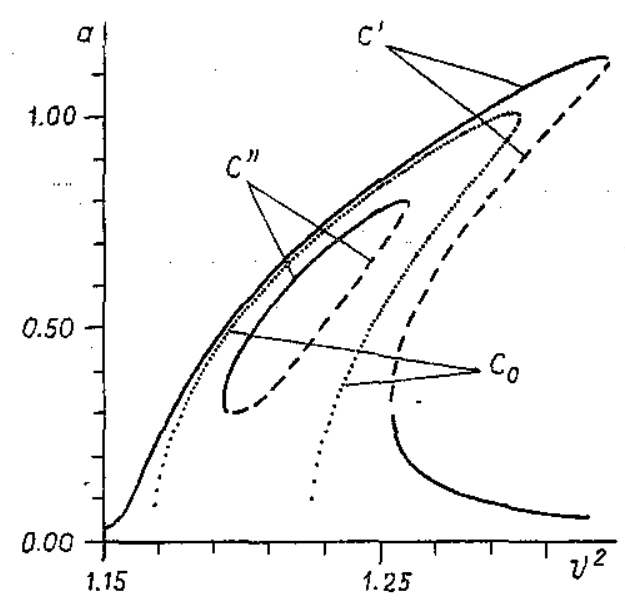

b)

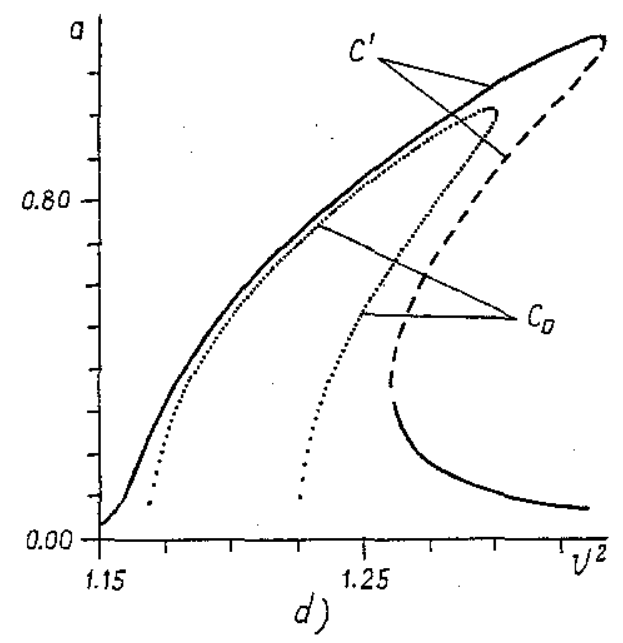

Fig. 5

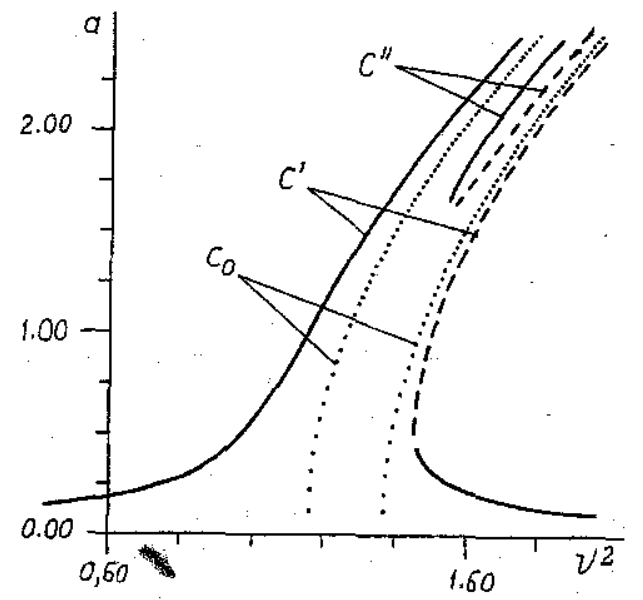

Fig. 6 
Varying $\delta$, the compatible point $I_{*}$ moves along $C_{0}$. In the case of the loop, as $a_{*}^{2}$ becomes less than $a_{c}^{2}$, the loop either disappears or changes into an oval. On the contrary, in the cases of the oval as $a_{*}^{2}$ exceeds $a_{c}^{2}$, the critical point $I_{*}$ appears.

In Figs $7(\mathrm{a}, \mathrm{b})$ for $h=0.09 ; \frac{b}{4} q^{2}=0.1 ; \frac{3}{4} \beta=0.1, p=0.03$ the loops $b, c, d, e, f, g, h$ correspond to $\delta=\frac{3 \pi}{4} \approx 2.35619 ; 2.32 ; 2.2519 ; 2.24 ; 2.4 ; 2.41936 ; 2.44$ respectively.

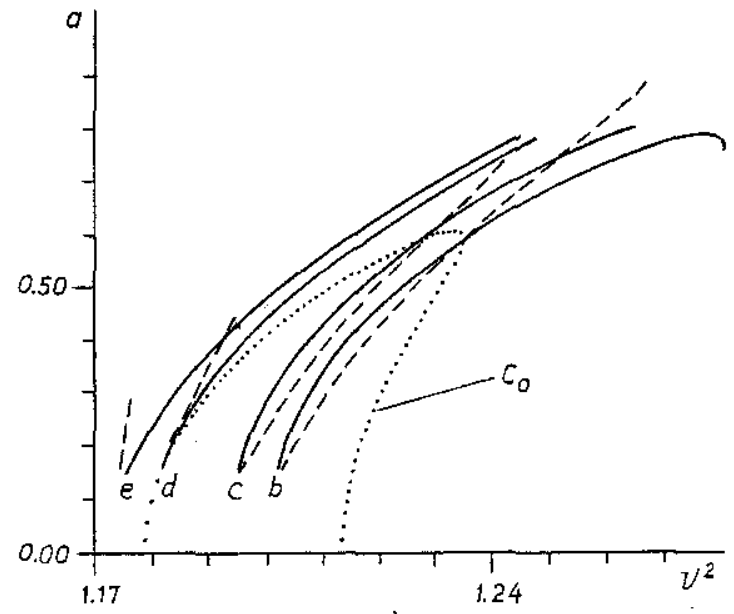

a)

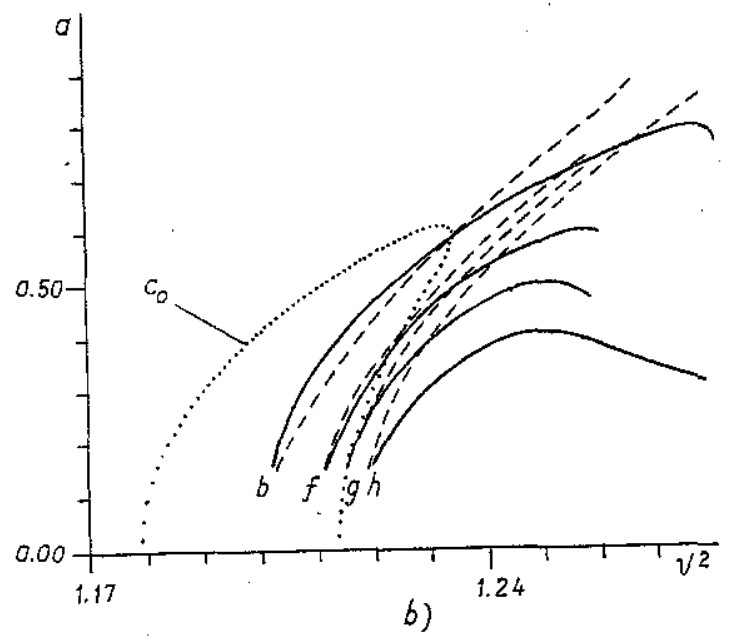

b)

Fig. 7

To illustrate the transformation of an oval into a loop, in Figs $8(a, b, c, d)$ for $h=0.0877$; $\frac{b}{4} q^{2}=0.1 \frac{3}{4} \beta=0.1 ; p=0.005$, the resonance curves have been drawn for $\delta=\frac{2 \pi}{3} \approx 2.09439 ; 2.15 ;$ $2.25 ; \frac{3 \pi}{4} \approx 2.35619$ respectively.

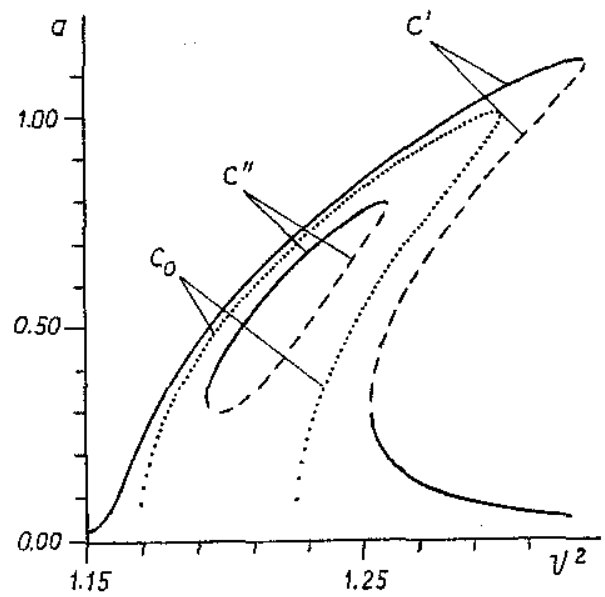

(1)

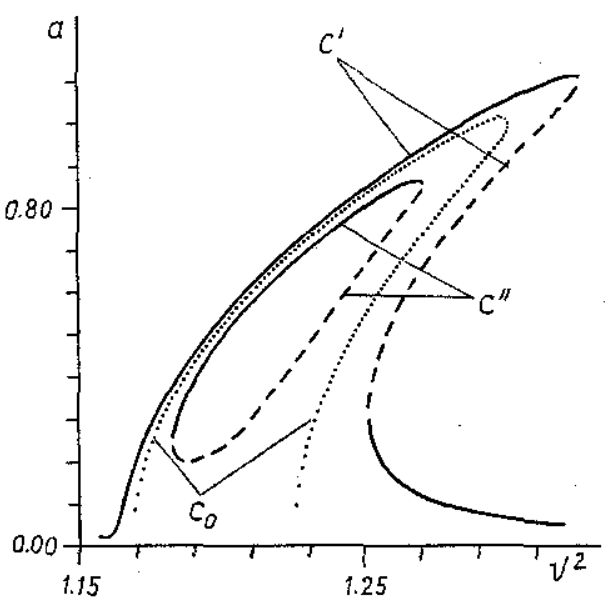

b) 

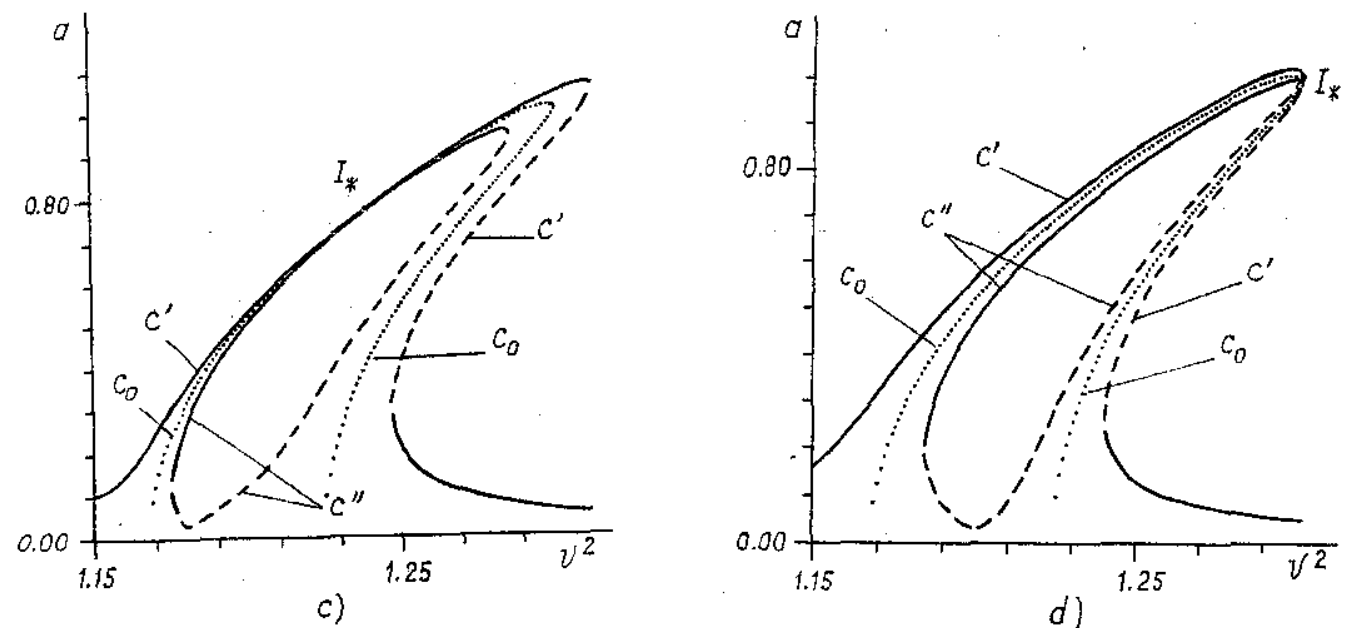

d)

Fig. 8

\section{Stability conditions}

Since $h_{0}>0$, then $a_{1} \rightarrow 0$, therefore we have only to study the stability character of the mode $y$. For this purpose, we use the variational equations

$$
(\delta a)^{*}=\frac{\varepsilon \nu}{2} \frac{\partial f_{0}}{\partial a}(\delta a)+\frac{\varepsilon \nu}{2} \frac{\partial f_{0}}{\partial \psi}(\delta \psi), \quad a(\delta \psi)^{*}=\frac{\varepsilon \nu}{2} \frac{\partial g_{0}}{\partial a}(\delta a)+\frac{\varepsilon \nu}{2} \frac{\partial g_{0}}{\partial \psi}(\delta \psi)
$$

where $(\delta a)(\delta \psi)$ are small perturbations of $a, \psi$ respectively.

The characteristic equation is of the form:

$$
a \rho^{2}+\frac{\varepsilon \nu}{2} S_{1} \rho+\frac{\varepsilon^{2} \nu^{2}}{4} S_{2}=0
$$

and sufficient conditions for asymptotic stability are:

$$
S_{1}=a \frac{\partial f_{0}}{\partial a}+\frac{\partial g_{0}}{\partial \psi}>0, \quad S_{2}=\frac{\partial f_{0}}{\partial a} \frac{\partial g_{0}}{\partial \psi}-\frac{\partial f_{0}}{\partial \psi} \frac{\partial g_{0}}{\partial a}>0
$$

The first stability condition is satisfied for the system with damping

$$
S_{1}=2 a \nu h>0 \text { i.e. } h>0 .
$$

Regarding to (1.5), the second stability condition can be written as:

$$
S_{2}=\frac{\partial f}{\partial a} \frac{\partial g}{\partial \psi}-\frac{\partial f}{\partial \psi} \frac{\partial g}{\partial a}>0
$$

For ordinary part $C_{1},(5.5)$ can be replaced by:

$$
\frac{1}{D_{0}} \cdot \frac{\partial W}{\partial a}>0
$$


This compact form can easily be used to determine ordinary stable portions of the resonance curve bounded by vertical tangents.

The stability of the critical nodal representing point $I_{*}$ can directly be deduced from that of the ordinary portion considered as containing $I_{*}$.

In the figures presented above heavy (broken) lines correspond to stable (unstable) stationary oscillations.

\section{Conclusion}

The interaction between forced and parametric oscillation in a system with two degrees of freedom has been examined. Critical singular points are used to classify different forms of the resonance curve. If the indirectly - excited parametric oscillation is not very intense, the resonance curve consists of two branches either joined at the critical representing point or separated by the critical region; correspondingly, the resonance curve has either a loop or an oval.

This publication is completed with financial support from the National Basic Research Programme in Natural Sciences

\section{References}

1. Mitropolskii Yu. A., Nguyen Van Dao. Applied asymptotic methods in nonlinear oscillations. Ukrainian Academy of Sciences, National centre for Natural Sciences and Technology of Vietnam, Kiev-Hanoi, 1994.

2. Nguyen Van Dinh. Stationary oscillations in critical cases (in Vietnamese) Journal of Mechanics NCNST of Vietnam T. XVIII, 1996, No 2, 13-19.

Received January \$, 1997

\section{TUOONG TÁC CƯỡG BỨC - THÔNG SỐ TRONG MộT HỆ HAI BẬC TỰ DO}

Xét tương tác giữa dao động thông số và cưỡng bức trong một hệ hai bậc tự do. Điểm kỳ dị tới hạn được dùng để phân loại dạng đường cộng hương. Khi dạo động thông số do kích động gián tiếp gây ra đủ mạnh (không quá mạnh), đường cộng hưởng gồm hai nhánh hoặc nối với nhau tại điểm kỳ dị tới hạn hoặc tách biệt nhau bơi miền tới hạn; tương ứng đường cộng hướng có một vòng thăt hoặc có nhánh bầu dục. 